\section{Nuclear Size in Premalignant Conditions of the Cervix Uteri}

Prevrous investigations have shown that according to their modal DNA content uterine and other tumours in man fall into two fairly discrete ploidy-groups ${ }^{1,2}$, and follow-up investigations in patients with uterine tumours suggest that the ploidy-group may be of prognostic significance $e^{3,4}$. From the size of the nuclei in histological sections of carcinoma of the cervix, it has been possible to make a correct estimate of the ploidy-group of the tumour in the majority of cases ; based on a large series of sections, 50 per cent of tumours were found to have predominantly small nuclei (corresponding to a neardiploid DNA content) and 50 per cent predominantly large nuclei (corresponding to a near-tetraploid DNA content) ${ }^{5}$. It would appear that a study of nuclear size in conditions commonly regarded as precursors of malignant disease in the cervix uteri might be of interest, and could possibly throw light on the relationship between the chromosomal changes, which appear to be a constant feature (though variable in extent) of human malignancy, and the apparently stepwise transformation of normal to malignant cervical epithelium.

Through the courtesy of Dr. A. Glücksmann, I have recently had the opportunity of looking through sections of basal-cell hyperplasia, carcinoma in situ and microcarcinoma of the cervix. The results are shown in Table 1. The majority of the sections were of material removed at conization of the cervix from patients with carcinoma in situ, discovered on routine cytological screening. Many of these patients were from ante-natal clinics; the mean age was $36 \cdot 7$ years, and the range $22-81$ years. Nuclear size was assessed as described for invasive tumours ${ }^{5}$, measurements being made on basal, parabasal and transitional cells; allowance was made for the increase in nuclear size during differentiation, as between the spindle-shaped basal-cell nuclei and the nearly spherical transitional-cell nuclei, an increase which, of course, is unrelated to any change in ploidy (tumours were placed in the 'large-nucleus' category if more than 20 per cent of the nuclei exceeded the following critical values : for transitional-cell nuclei, a diameter of $10 \mu$, or, if noncircular in outline, a product of the major and minor axes in microns of 100 , and for spindle-shaped nuclei of basalcell type, the minor axis of which was between $\frac{1}{3} \times$ and $\frac{1}{2} \times$ the major axis, a major axis of $13 \mu$ ). It was found that the carcinomas in situ, like invasive carcinomas, fell into two groups on the basis of their predominant nuclear size. The lesions were also classified histologically as squamous or basocolumnar (I thank Dr. Glücksmann for the histopathological assessments). Of 77 squamous lesions, 54 had large nuclei (Table 1). This is significantly different $(P<0.001)$ from the distribution for invasive squamous-cell carcinomas (401 out of 803 having large nuclei $\left.{ }^{5}\right)$. On the other hand, only 4 out of 10 basocolumnar lesions had large nuclei. No relation between nuclear size and age was apparent. Most cases were consistent with regard to nuclear size throughout the lesion (an average of 2.4 slides per case showing carcinoma in situ were available for examination). However, 9 cases classified as predominantly 'small-nucleus' had some 'large-nucleus' areas, and 10 cases with predominantly large nuclei had some 'small-nucleus' areas.

The greater frequency of 'large-nucleus' in situ carcinomas as compared with invasive carcinomas raises some interesting possibilities. Perhaps carcinoma in situ with large nuclei progresses less often or more slowly to invasive cancer; or perhaps there may be a change from a large to a small-nucleated type when invasion occurs. It may be relevant that some invasive tumours having a near-tetraploid modal DNA content have a minority of near-diploid cells, as shown by the presence of a small secondary mode in the diploid region or of metaphases with near-diploid ehromosome numbers. It is possible that in some carcinomas in situ the majority of cells may
Table 1. Nuclear Size in 18 Cases OF Basal-Ceil Hyperplasia, 87 Cases of Carcinoma in situ AND 24 CASES OF MIOROCARCINOMA OF THE Cervix UTERI
Small nuclei Large nuclei Total

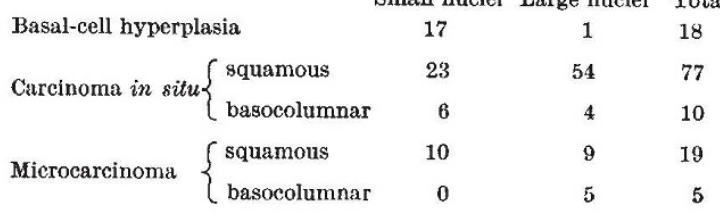

be near-tetraploid, but there may be a few near-diploid cells which become predominant when invasion occurs. It is of interest that one lesion classified as 'large-nucleus' showed a deep prolongation in which the nuclei were mostly small. Although in invasive tumours aneuploidy is the rule, the findings in carcinoma in situ, while indicating varying degrees of polyploidy, are compatible with normal chromosome complements. It is of interest that the chromosome counts of Spriggs, Boddington and Clarke ${ }^{\theta}$ on 5 cases of carcinoma in situ showed aneuploidy in some instances, but only apparently normal tetraploid cells in 2 cases. It was noted in the present work that in the 'small-nucleus' lesions there was a substantial minority of intimately mixed cells having large nuclei, comparable to what has been found in small-nucleus invasive tumours. A sharp transition to apparently normal epithelium was noted in many cases; this was especially apparent when the basal layer of 'large-nucleus' lesions was followed to the junction with normal epithelium.

Sections were examined from 24 cases of microcarcinoma (defined by Glücksmann and Cherry ${ }^{7}$ as carcinoma in situ with early or questionable invasion of the cervical stroma, and carcinoma in situ with definite invasion of the cervical stroma but lacking in ability to grow in xenotopic sites). The mean age of the patients was 44.4 years. The results (Table 1) do not suggest such a preponderance of large. nucleus types as for carcinoma in situ, but rather a distribution similar to that found in invasive carcinoma; however, owing to the small number of cases the results are not statistically significant.

Finally, sections from 18 cases diagnosed as basal-cell hyperplasia (mean age, 35.5 years) were examined; only one had predominantiy large nuclei, although two others which had predominantly small nuclei also had some areas showing mainly large nuclei. Of 17 having small nuclei, 9 showed very little variation in size, comparable to that found in normal epithelium, while the remainder showed a proportion of cells with large nuclei similar to that found in small-nucleus carcinoma in situ or invasive carcinoma.

The findings suggest that either polyploidy with normal tetraploid chromosome complements or aneuploidy with near-tetraploid complements is of frequent but variable occurrence in carcinoma in situ, and may occasionally be prominent in even earlier stages of carcinogenesis. The question as to whether nuclear size (or DNA content) may be of prognostic value in carcinoma in situ would seem worthy of investigation.

I thank Mr. Stanley Way for supplying the material on which this work was based, and Dr. Alfred Glücksmann for his assistance and advice. This work was supported by a grant from the British Empire Cancer Campaign.

N. B. AtKIN

Department of Cancer Research,

Mount Vernon Hospital,

Northwood,

Middlesex.

' Atkin, N. B., Richards, B. M., and Ross, A. J., Brit. J. Cancer, 13, 773 (1959). ${ }^{2}$ Atkin, N. B., in Human Chromosome Abnormalities, edit. by Davidson,
W. M., and Robertson Smith, D., 135 (Staples Press, London, 1961).

a Atkin, N. B., and Richards, B. M., Brit. Med. J., it, 1445 (1962).

4Atkin, N. B., Brit. J. Radiol., 37, 213 (1964).

Atkin, N. B. (in preparation).

- Spriggs, A. I., Boddington, M. M., and Clarke, C. M., Lancet, i, 1383 (1962).

${ }^{7}$ Glücksmann, A., and Cherry, C. P. (personal communication). 